\title{
Quasiparticle Recombination Time of Superconducting Tin Films in a Parallel Magnetic Field
}

\author{
K. Holdik, M. Welte, and W. Eisenmenger \\ Physikalisches Institut der Universität Stuttgart, Stuttgart, West Germany
}

(Received October 24, 1983; revised August 10, 1984)

The influence of a parallel magnetic field on the quasiparticle recombination time of superconducting tin tunneling junctions has been determined experimentally. The results are compared with the predictions of a theoretical model that takes into account the modification of the quasiparticle density of states by the applied magnetic field.

\section{INTRODUCTION}

If the quasiparticle concentration in a superconductor is enhanced compared to the thermal value, the corresponding nonequilibrium relaxes with a characteristic time constant usually called $\tau_{\text {eff }}$. This quasiparticle recombination time has been investigated in several theoretical and experimental studies. ${ }^{1-16}$ The essential results are that quasiparticles excited to the gap energy $\Delta$ decay via pair recombination and emission of phonons of energy $2 \Delta$. The intrinsic recombination time $\tau_{\mathrm{R}}$ (i.e., the lifetime of a quasiparticle) is determined by the strength of the electron-phonon interaction and the quasiparticle concentration in the superconductor. Due to these recombination processes, the creation of excess quasiparticles in a superconductor is accompanied by an enhancement of the density of phonons with energy $\Omega \geqslant 2 \Delta$. Further, with films of strong coupling superconductors such as $\mathrm{Sn}$ or $\mathrm{Pb}$, the mean free path $\Lambda$ of the $2 \Delta$ phonons is comparable to or less than the film thickness; therefore the $2 \Delta$ phonons are trapped in the superconductor by pair-breaking reabsorption. This phonon trapping increases the experimentally observable quasiparticle time constant $\tau_{\text {eff }}$ significantly compared to the intrinsic value $\tau_{\mathrm{R}}{ }^{6}{ }^{6}$

Experimentally superconducting tunneling junctions have been found to be an ideal instrument for studying the effective quasiparticle decay constant, because the quasiparticle density can be deduced directly from 
the thermal tunneling current (bias voltage $V<2 \Delta / e$ ). Quasiparticles can be created either by tunneling injection or by irradiation with photons or high energy phonons. The excess quasiparticle population may be probed either in steady state or in time-resolved decay measurements.

In this paper the influence of a parallel magnetic field on the decay time in tin tunneling junctions is determined. We find a reduction of the decay time with increasing field. In order to interpret these results, a theoretical model is developed that takes into account the well-known modifications of the electronic excitation spectrum of a superconductor by the magnetic field.

\section{THEORY}

Tewordt ${ }^{3}$ treated the lifetime $\tau_{\mathrm{R}}$ of a quasiparticle of energy $E$ with respect to recombination process (magnetic field $H=0$ )

$$
\begin{aligned}
\tau_{\mathrm{R}}^{-1}(E)= & C f^{-1}(-E) \int_{\Delta}^{\infty} d E^{\prime}\left(E^{\prime}+E\right)^{2} N\left(E^{\prime}\right) f\left(E^{\prime}\right) \\
& \times\left[1+\Delta^{2} /\left(E E^{\prime}\right)\right]\left[1+B\left(E^{\prime}+E\right)\right]
\end{aligned}
$$

Here $C$ is a parameter describing the strength of the electron-phonon interaction, $N(E)=4 N_{0} E /\left(E^{2}-\Delta^{2}\right)^{0.5}(E>\Delta)$ is the BCS density of states, and $f$ and $B$ are the Fermi and the Bose-Einstein distribution functions. This formula is strictly valid only in the limit of the jellium model, neglecting real metal effects such as Umklapp processes and nonspherical Fermi surface. ${ }^{4,10}$ Furthermore, the electron mean free path $l$ and the wave vector $q$ of the $2 \Delta$ phonons must satisfy the relation

$$
q l>1
$$

If $\beta=\Delta / k T \gg 1$, the following approximation is valid for Eq. (1), describing the temperature dependence:

$$
\tau_{\mathrm{R}}^{-1}(\Delta)=4 N_{0} C(2 \Delta)^{3}(2 \pi / \beta)^{0.5} e^{-\beta}
$$

(Here and in the following we exclude the temperature range very close to $T_{c}$, where the order parameter relaxation time diverges. ${ }^{17,18}$ )

If the total thickness $d_{1}+d_{2}$ of the two-junction films is greater than the mean free path $\Lambda$ of the $2 \Delta$ phonons, the experimentally observable decay time is ${ }^{13,14}$

$$
\tau_{\text {eff }}=4 \tau_{\mathrm{R}}\left(d_{1}+d_{2}\right) /\left[\Lambda\left(\bar{T}_{1}+\bar{T}_{2}\right)\right]
$$

where $\bar{T}_{1}$ and $\bar{T}_{2}$ are the phonon transmission coefficients of the two boundaries (junction-substrate and junction-helium or junction-vacuum) 
averaged over the angles of incidence. For helium-covered tin diodes it has been verified experimentally that $\tau_{\text {eff }}$ is proportional to the total film thickness $d_{1}+d_{2}$, according to Eq. (4), in the thickness range $3000 \leqslant d_{1}+d_{2} \leqslant$ $9000 \AA . .^{15}$

A generalization of Eq. (1) for the case of an applied parallel magnetic field $H$ can be obtained in the following way: If a magnetic field is applied, the density of states is altered. According to the calculations of Skalski et $a l^{19}$ (also see Refs. 20 and 21), the new expression is

$$
N(E)=4 N_{0}\left|(E / \Delta) Z^{-0.5} \operatorname{Im}[1 /(X-Z)]\right|
$$

Here $Z$ is a parameter related to the magnetic field $H$ and the order parameter $\Delta$ by

$$
\begin{aligned}
\ln \left(\Delta / \Delta_{0}\right) & =-(\pi / 4) Z^{1.5} \\
\left(H / H_{c}\right)^{2} & =2 Z^{1.5}\left(\Delta / \Delta_{0}\right)
\end{aligned}
$$

and $X$ is a complex root of the quartic equation

$$
X^{4}-2 Z X^{3}+\left[(E / \Delta)^{2} / Z-1 / Z+Z^{2}\right] X^{2}+2 X-Z=0
$$

Thereby, we suppose $T / T_{c} \ll 1$. Furthermore, Eq. (6) is only valid for $0 \leqslant Z<1$; i.e., we consider only the nongapless regime, where a finite minimum excitation energy $E_{0}$ exists:

$$
E_{0}=\Delta(1-Z)^{1.5}
$$

Levine $^{21}$ found that Eqs. (5)-(9) may be applied to superconducting films of thickness $d$, electronic mean free path $l$, and BCS coherence length $\xi_{0}$ if the following relations hold:

$$
\begin{gathered}
l \ll \xi_{0} \\
l\left(l / \xi_{0}\right) \ll d \ll\left(l \xi_{0}\right)^{0.5}
\end{gathered}
$$

Here the right inequality ensures that $\Delta$ does not vary across the film thickness. Theoretical and experimental investigations of the influence of a finite mean free path ${ }^{22,23}$ yielded the result that up to a ratio $l / \xi_{0} \leqslant 0.3$ the density of states given by Eq. (5) is a good approximation.

In order to avoid screening effects, it is desirable to choose a film thickness smaller than the magnetic penetration depth $\lambda_{\text {eff }}$, i.e.,

$$
d \approx \lambda_{\text {eff }}=\lambda_{\mathrm{L}}\left(1+\xi_{0} / l\right)^{0.5}
$$

where $\lambda_{\mathrm{L}}$ is the London penetration depth. Especially if

$$
d>\sqrt{5} \lambda_{\mathrm{eff}}
$$


the film undergoes a first-order phase transition in an applied magnetic field, as in the transition of a bulk superconductor.

The relevant parameters for tin are $\xi_{0}=2300 \AA$ and $\lambda_{\mathrm{L}}=350 \AA .^{20} \mathrm{In}$ order to determine $l$ from the normal resistance $\rho$ we used the relation $\rho l=10^{-11} \Omega \mathrm{cm}^{2},{ }^{20}$ resulting in values ranging from $l=200$ to $1200 \AA$ for our samples.

\section{EXPERIMENTAL ARRANGEMENT AND PROCEDURE}

In our experiment the time constant $\tau_{\text {eff }}$ was determined by analyzing the experimental signal decay time of tin tunneling junctions used as detectors for pulses of high frequency phonons. ${ }^{11,25} \mathrm{~A}$ second tunneling junction served as phonon pulse generator. The two tin junctions were evaporated onto opposite faces of a $\langle 100\rangle$-oriented silicon crystal $(7 \mathrm{~mm}$ thick, $15 \mathrm{~mm}$ diameter). In this orientation the transverse phonon signal is favored by phonon focusing by more than a factor of ten. ${ }^{26}$ Due to this and to the high ratio of diameter to crystal thickness, the relative strength of the side-wall-reflected phonon signal is strongly reduced, thus facilitating the determination of the pulse decay time of the direct ballistic phonon signal.

The entire arrangement is mounted in a sample chamber that allows measurements with diodes either covered by helium or under vacuum conditions. In most of the experiments the sample cell was filled with gaseous $\mathrm{He}$ at $\sim 0.1$ Torr. Regarding the influence of the surface covering on the effective time constant $\tau_{\text {eff }}$, these conditions are identical to an immersion in bulk helium. On the other hand, under these conditions frozen flux may be removed from the tin films by heating only the substrate crystal over $T_{c}$. The cooling by the surrounding helium bath provided temperatures down to $1.0 \mathrm{~K}$. A magnetic field was applied by a superconducting solenoid. The superconducting films of the tunneling junctions and the magnetic field direction could be aligned parallel prior to the lifetime measurement. This alignment was achieved by minimizing the effect of frozen flux, which can be observed in the current-voltage characteristics of the junctions.

Nevertheless, with magnetic fields exceeding $600 \mathrm{G}$, frozen flux could not be avoided.

The principles of fast pulse detection with superconducting tunneling junctions are given in Ref. 25 . In our arrangement the overall electronic time constant of the detection system was $\leqslant 80 \mathrm{nsec}$, determined mainly by the pulse transformer $(60 \mathrm{nsec})$ and the pulse amplifier $(30 \mathrm{nsec})$. The error of the absolute value of the time constant determined in this way is estimated to be $\pm 15 \%$, but the relative errors of time constants obtained in the same measurement remained below $5 \%$. 
TABLE I

Tunneling Junction Data

\begin{tabular}{lcccc}
\hline \multicolumn{1}{c}{ Sample } & 1A & 1B & 2A & 2B \\
\hline Total thickness $d_{1}+d_{2}, \AA$ & 2200 & 6000 & 4280 & 4200 \\
$\begin{array}{l}\text { Energy gap } 2 \Delta, \mathrm{meV} \\
\text { Dynamical resistance } d V / d I\end{array}$ & 1.19 & 1.18 & 1.14 & 1.20 \\
$\quad$ at detector bias point, $\mathrm{m} \Omega$ & 255 & 277 & 1870 & 700 \\
$\begin{array}{l}\text { Critical magnetic field } H_{c}, \mathrm{Oe} \\
\text { Nature of the phase transition }\end{array}$ & 800 & 363 & 505 & 553 \\
$\quad$ at $H_{c}$ & Second order & First order & First order & First order \\
\hline
\end{tabular}

Table I summarizes the data for the tunneling junctions. From the normal resistance measured on identically prepared strips we derived a value of $l=200-1200 \AA$ for the electronic mean free path.

Only the thinnest film, sample $1 \mathrm{~A}$, with a thickness of roughly $1000 \AA$, just meets the condition for a quantitative comparison with the theoretical results described earlier. The other films are thicker and within a regime where the gap parameter as well as the phonon absorption and quasiparticle recombination rates have an inhomogeneous spatial distribution within the films. Since we did not use substrate cooling during the evaporation of the tin films, the microcrystalline film structure set a lower thickness limit of $1000 \AA$.

\section{EXPERIMENTAL RESULTS AND DISCUSSION}

The observed magnetic field dependence of the recombination time $\tau_{\text {eff }}$ for different samples $(T \approx 1 \mathrm{~K})$ is shown in Figs. 1-3. In the case of a thin junction (sample 1A; see Table I) the decrease of the time constant can be fitted to a linear magnetic field dependence as shown in Fig. 1, curves a and $b$, whereas in the case of junctions with thicker films (1B, 2A, 2B) three distinct magnetic field dependence ranges are observed, as shown in Fig. 1, curves $\mathrm{c}$ and d, and Figs. 2 and 3: at low fields, $\tau_{\text {eff }}$ is only weakly field dependent, followed by an apporximately linear reduction with 1.2$1.6 \mathrm{nsec} / \mathrm{Oe}$. Finally, at fields exceeding $\sim 300$ Oe the time constant remains nearly field independent at a value of $\tau_{\text {eff }} \approx 200 \mathrm{nsec}$, which is significantly higher than the time constant of the electronic detection system. Because of the rapid decrease of the time constant and the corresponding loss of detector sensitivity, measurements at higher termperatures could only be performed with junction $2 \mathrm{~A}$, due to its high dynamical resistance (Table I).

At higher temperatures an enhancement of the effective time constant with increasing field also could be observed (Fig. 4). A positive slope of 


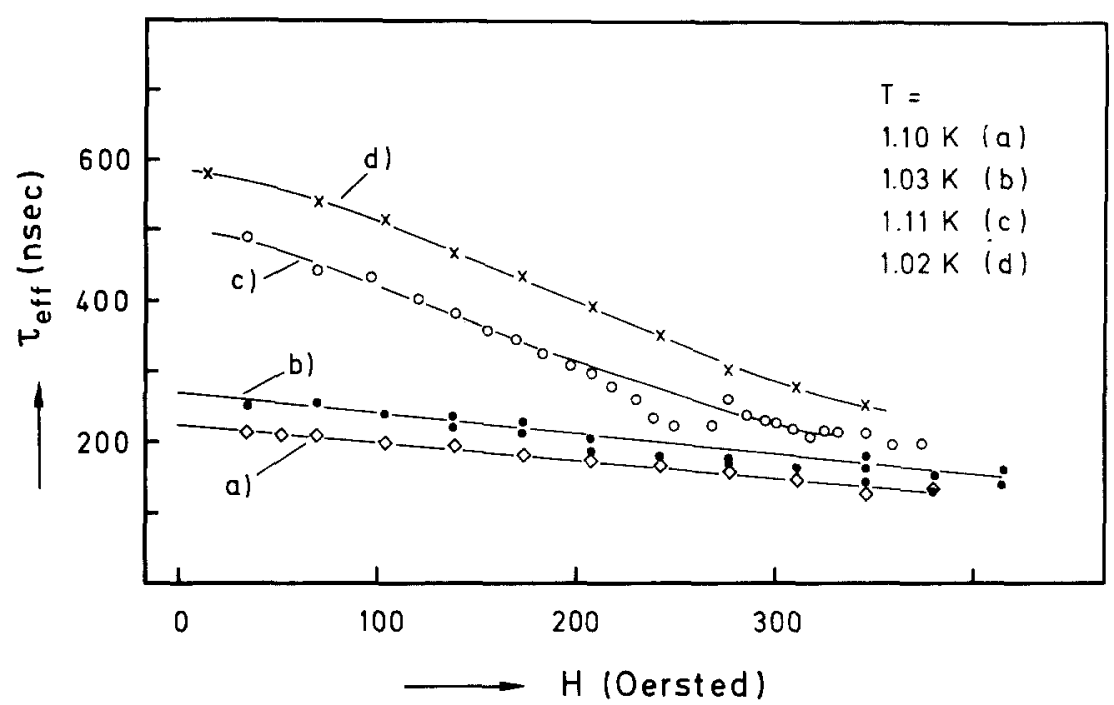

Fig. 1. Magnetic field dependence of the observed effective quasiparticle lifetimes $\tau_{\text {eff }}$ in $(\mathrm{a}, \mathrm{b})$ sample 1A and $(\mathrm{c}, \mathrm{d})$ sample $\mathrm{B}$. Curve a was determined in vacuum; in all other cases the sample chamber was filled with helium gas.

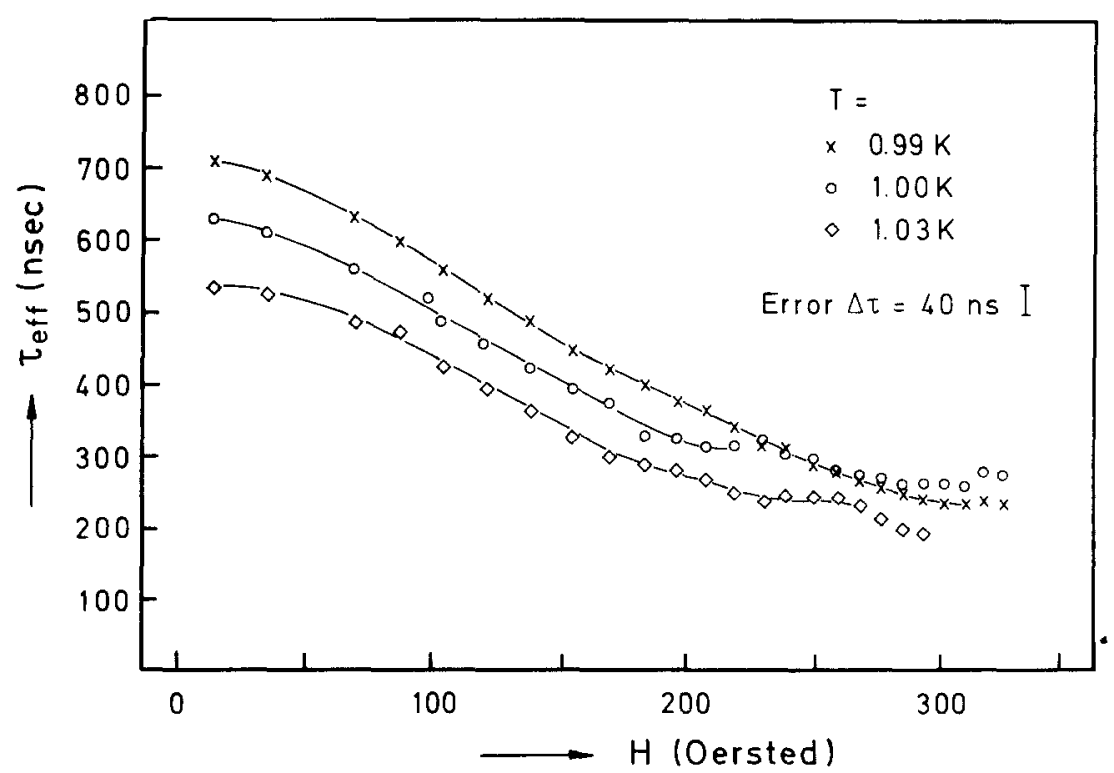

Fig. 2. Magnetic field dependence of the effective quasiparticle lifetime $\tau_{\text {eff }}$ in sample $2 \mathrm{~A}$. The sample chamber was filled with helium gas. 


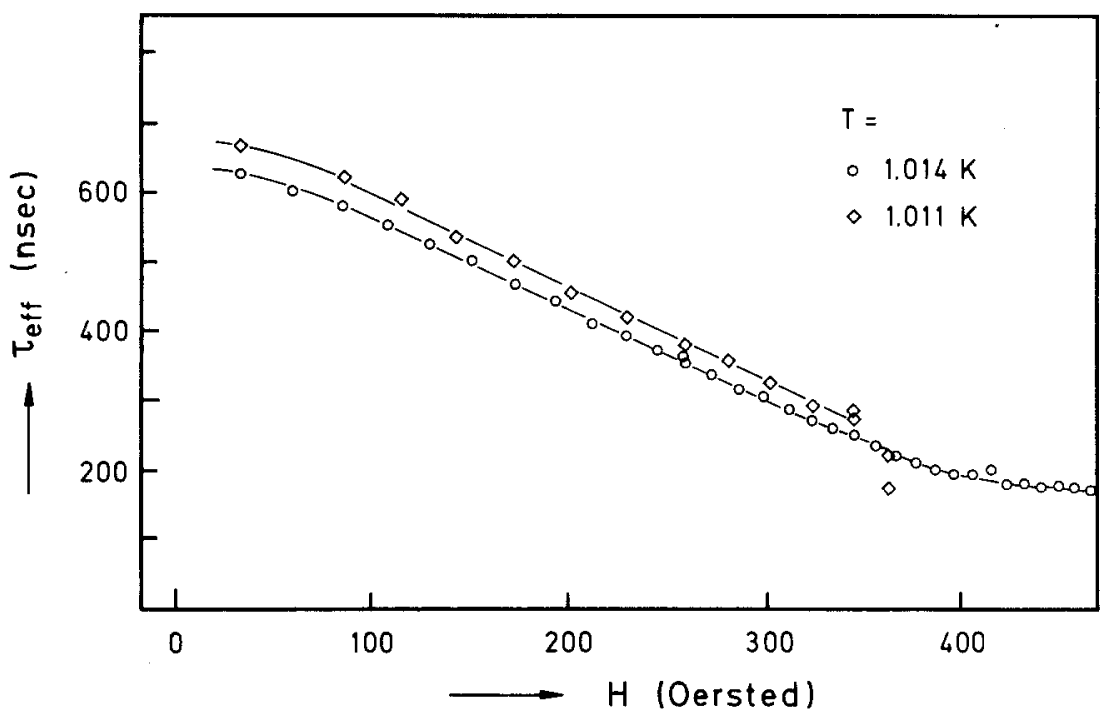

Fig. 3. Magnetic field dependence of the effective quasiparticle lifetime $\tau_{\text {eff }}$ in sample 2B. The sample chamber was filled with helium gas.

$d \tau / d H$ was further obtained in the high field region of thick film junctions (Fig. 5). In this case we found that this change in slope is correlated with the additional injection current resulting if the field-dependent spectral energy gap $2 E_{0}(H)$ is smaller than the detector bias voltage $V_{D}$. These effects show that in the presence of quasiparticles that may be either

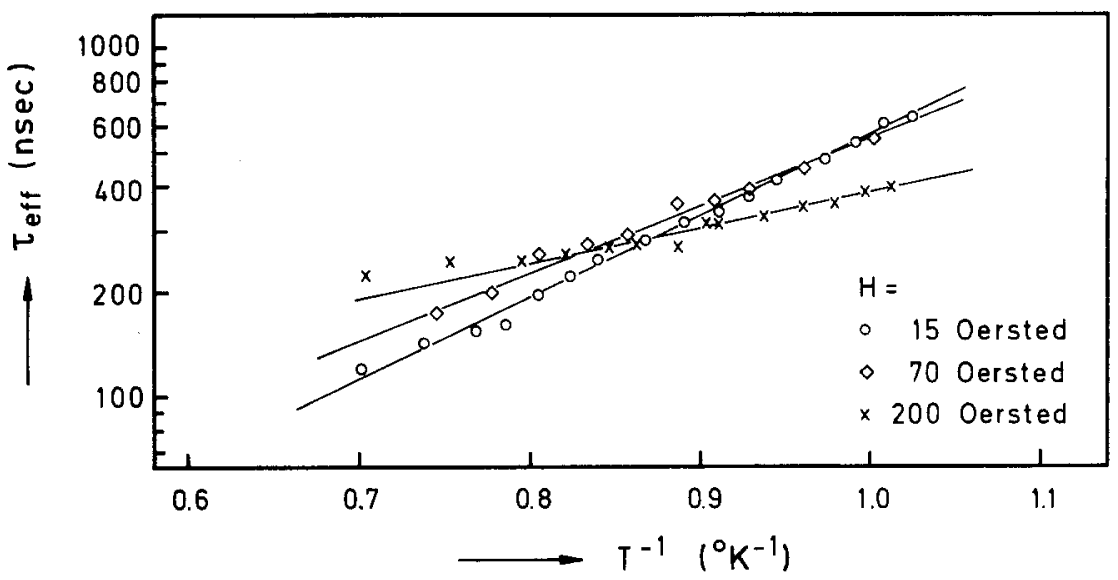

Fig. 4. Temperature dependence of the effective quasiparticle lifetime in sample $2 \mathrm{~A}$ at constant magnetic field. The solid lines correspond to an exponential law exp $(-\Delta / k T)$ with $\Delta=0.46,0.39$, and $0.2 \mathrm{meV}$. 


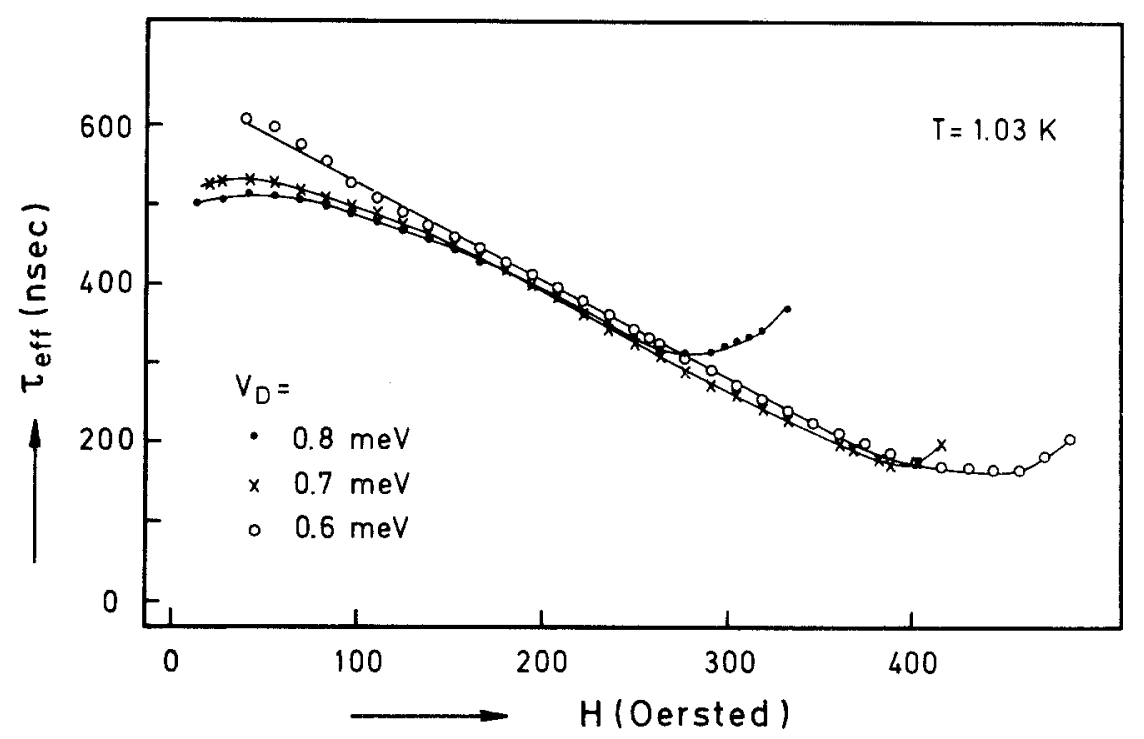

Fig. 5. The observed effective quasiparticle lifetime in junction $2 \mathrm{~B}$. In this experiment the detector bias voltage $V_{D}$ is automatically fixed by a feedback loop. The injection of quasiparticles begins if the field-dependent spectral energy gap $2 E_{0}(H)$ is less than $V_{D}$. Measurements at higher fields are impossible because the detector sensitivity is reduced by the decrease of the dynamical resistance.

thermally excited or produced by injection, the magnetic field can exhibit a delaying influence on the recombination process.

The results of numerical calculations according to Eqs. (1)-(8) are given in Fig. 6. Qualitatively, the experimentally observed slope is reproduced and it is further predicted that the relative field-induced reduction of the time constant is stronger at low temperature.

A quantitative comparison with the experiment seems to be reasonable only in the case of the thin film junction 1A. If the linear range of the field dependence is described by a relation of the form $\tau(H)=$ $A \tau(H=0)\left[1-B\left(H / H_{c}\right)\right]$, the $A$ and $B$ parameters obtained by fitting the experimental data are $A_{\text {exp }} \approx 1.0$ and $B_{\text {exp }} \approx 0.9$, whereas the model calculations predict $A_{\text {calc }}=1.2$ and $B_{\text {calc }}=1.5$. This rough agreement appears satisfactory in view of the fact that the theoretical film thickness condition is just met by sample 1A. In this case remaining inhomogeneities of the order parameter may cause the observed deviations.

In this respect it appears remarkable that the results for the other samples with increased film thickness still exhibit qualitative agreement with theory (see Fig. 6). This may be explained as follows: assuming the gap parameter to be lower at the surface than in the bulk of the superconduct- 


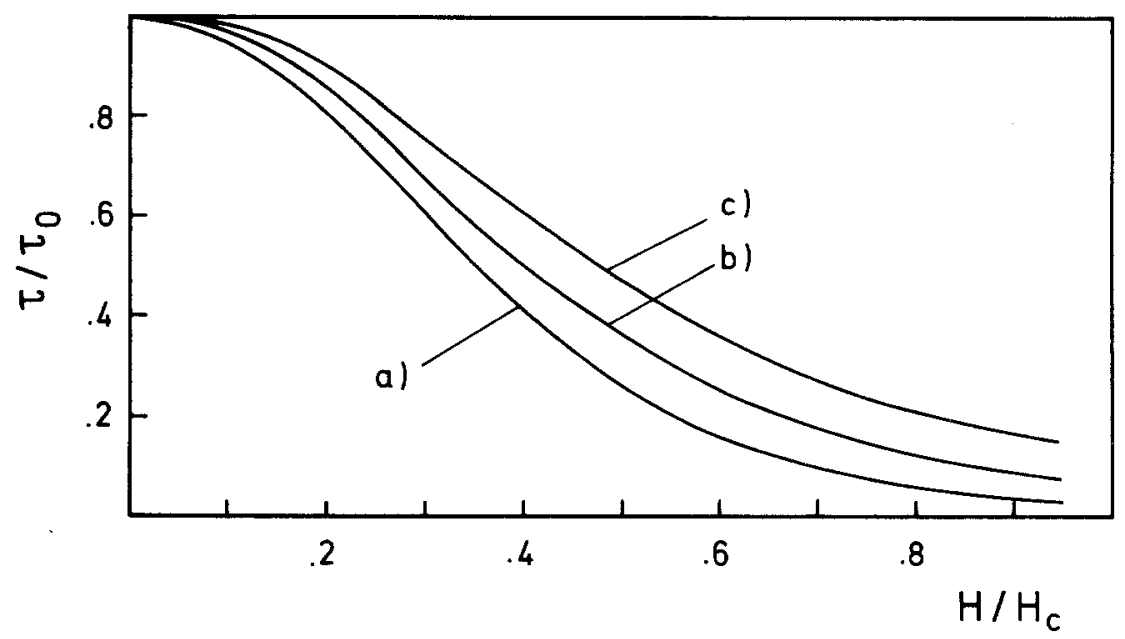

Fig. 6. Calculated ratio $\tau(H) / \tau(0)$ for three temperatures: $\Delta / k T$ equals (a) 7, (b) 6 , (c) 5 .

ing film, the quasiparticle phonon system in reemission and reabsorption tends to undergo an oriented diffusion to the lower gap region during relaxation. Thus, more of them should recombine at a surface region where the excitation energy calculated in Eq. (9) holds although screening effects are apparent. Thus, leaving the thin film regime [Eq. (11)], the dependence of the quasiparticle lifetime on magnetic field should not change radically simply by the dominating influence of recombination in the film boundary regime.

\section{CONCLUSION}

Summarizing, it may be said that the general features of the experimentally determined behavior of the quasiparticle recombination time in a magnetic field can be interpreted by an extension of the current theories on the quasiparticle-phonon interaction in superconductors. Nevertheless, future experimental work may concentrate on thinner films of homogeneous order parameter and the interesting range of higher temperatures, whereas theoretical work on thicker films taking account of the inhomogeneous order parameter and phonon reemission and reabsorption rates would be very interesting. In addition, one important conclusion can be drawn from the above work: when tin tunneling junctions are used as phonon and heat pulse detectors, often a magnetic field of up to 10 Oe must be applied in order to suppress the dc Josephson effect. From our results it follows that the sensitivity and the properties of the spectral response are not affected in this magnetic field regime. 


\section{ACKNOWLEDGMENTS}

We thank Dr. O. Koblinger for useful discussions during this work; we also thank G. Mayerhoeffer for the preparation of the substrate crystals.

\section{REFERENCES}

1. D. M. Ginsberg, Phys. Rev. Lett. 8, 204 (1962).

2. J. R. Schrieffer and D. M. Ginsberg, Phys. Rev. Lett. 8, 207 (1962).

3. L. Tewordt, Phys, Rev. 128, 12 (1962).

4. A. Rothwarf and M. Cohen, Phys. Rev. Lett. 130, 1401 (1963).

5. B. I. Miller and A. H. Dayem, Phys. Rev. Lett. 18, 1000 (1967).

6. A. Rothwarf and B. N. Taylor, Phys. Rev. Lett. 19, 27 (1967).

7. W. Eisenmenger, in Fachberichte der Physikertagung Berlin 1967, Deutsche Physikalische Gesellschaft, ed. (B. G. Teubner, Stuttgart, 1967), p. 88.

8. J. L. Levine and S. Y. Hsieh, Phys. Rev. Lett. 20, 994 (1968).

9. K. E. Gray, A. R. Long, and C. J. Adkins, Phil. Mag. 20, 273 (1969).

10. K. E. Gray, Phil. Mag 20, 267 (1969).

11. W. Eisenmenger, in Tunneling Phenomena in Solids, E. Burstein and S. Lundquist, eds. (Plenum Press, New York, 1969), p. 371.

12. A. Rothwarf, Phys. Rev. Lett. 23, 468 (1969).

13. H. J. Trumpp, K. Lassmann, and W. Eisenmenger, Phys. Lett. 41A, 431 (1972).

14. W. Eisenmenger, K. Lassmann, H. J. Trumpp, and R. Krauss, Appl. Phys. 11, 307 (1977).

15. W. Eisenmenger, K. Lassmann, H. J. Trumpp, and R. Krauss, Appl. Phys. 12, 163 (1977).

16. P. W. Epperlein, K. Lassmann, and W. Eisenmenger, Z. Phys. B 31, 377 (1978).

17. G. Lucas and M. J. Stephen, Phys. Rev. 154, 349 (1967).

18. A. Schmid and G. Schön, J. Low Temp. Phys. 20, 207 (1975).

19. S. Skalski, O. Betbeder-Matibet, and P. R. Weiss, Phys. Rev. 136, A1500 (1964).

20. P. Fulde, Phys. Rev. 137, A783 (1965).

21. J. L. Levine, Phys. Rev. 155, 373 (1967).

22. S. Strässler and P. Wyder, Phys. Rev. 158, 319 (1967).

23. J. Millstein and M. Tinkham, Phys. Rev. 158, 325 (1967).

24. W. J. Stocpol, Phys. Rev. B 14, 1045 (1967).

25. W. Eisenmenger, in Physical Acoustics, Vol. XII, P. W. Mason and R. N. Thurston, eds. (Academic Press, New York, 1976), p. 79.

26. D. Marx and W. Eisenmenger, Z. Phys. B 48, 277 (1982). 